\title{
Agrarökologische Aspekte - zum Wert nachhaltiger Beweidung
}

\author{
Anita Idel
}

Die Bedeutung nachhaltiger Beweidung für die Welternährung könnte kaum größer sein: Ihre Potenziale erstrecken sich auf das Klima und die Basisressourcen - Bodenfruchtbarkeit, Gewässerqualität, biologische Vielfalt - sowie die Tiergesundheit. Das betrifft die (weltweite Entstehung der) fruchtbaren Kornkammern ebenso wie Land, das nicht beackert werden kann, weil es zu steil, zu steinig oder zu nass ist.

Die Entwicklung des Ökosystems Weide begann bereits vor 60 Millionen Jahren: eine Ko-Evolution zweier Lebenspartner - Grasland und weidende Tiere. Aber seine Potenziale für nachhaltige Ressourcennutzung und Klimaentlastung werden in Wissenschaft, Politik und (medialer) Öffentlichkeit dramatisch unterschätzt - und weitgehend ignoriert.

Die Risiken für die Ernährung steigen; denn die landwirtschaftliche Produktion wird weiter auf Spezialisierung, Maximierung und Normierung ausgerichtet. Ob Pflanzen, Tiere oder Böden: So verkommt die Landwirtschaft - von Pestiziden über chemisch-synthetischen Stickstoffdünger bis zu Antibiotika - zunehmend zu einem Reparaturbetrieb, weshalb auch z. B. Bodenerosion und bedrohliche Resistenzen weiter zunehmen.

Geld wird viel mehr an diesem kranken Agrar- und Ernährungssystem verdient und nicht innerhalb nachhaltiger Kreislaufsysteme. Aber die Lösung liegt in einer Landwirtschaft mit resilienten Ökosystemen einschließlich gesunder Doppelnutzungsrinder auf artenreichen Weiden.

\section{Der Blick zurück}

\subsection{Wider die Geschichtsvergessenheit}

Fast 50 Jahre nach „Die Grenzen des Wachstums“ (Donella H. Meadows et al. 1972) und über ein Vierteljahrhundert nach der UN-Konferenz für Umwelt und Entwicklung (Rio-Konferenz 1992) gilt weiterhin die Maxime Wachstum, welche zunehmend zerstörerisch auf Landschaft, Tier und Mensch wirkt. Hunger und Mangelernährung sind Folge der Ressourcen 
verbrauchenden sowie verschmutzenden und zerstörenden Produktionsbedingungen, derweil immer noch mehr produziert wird. Aber das ist - auch angesichts der Vernichtung und Verschwendung von Lebensmitteln - nur ein scheinbarer Widerspruch. Denn benötigt wird nicht ein Mehr an Nahrung oder Kalorien. Erforderlich sind gesunde Lebensmittel, von deren Produktion die Bäuerinnen und Bauern sowie Betreiber regionaler Verarbeitungsbetriebe leben können. Das Drama der Entwicklung der letzten Jahrzehnte belegen zwei UN-Berichte: 2014 waren weltweit 570 Millionen Höfe Familienbetriebe und damit das vorherrschende Modell der Landwirtschaft. Mit der Produktion von 80 Prozent aller weltweit konsumierten Lebensmittel bilden sie das Rückgrat der Welternährung (FAO 2014, 2). Gleichzeitig zählt dazu ein Großteil der Hungernden - verarmte Kleinbäuerinnen und Kleinbauern (Beverly McIntyre et al. [Hg.] 2009, 8).

\subsection{Der Weg zur Exportorientierung für tierische Produkte}

Seit den 1970er-Jahren wurden Agrar-Forschung, -Ausbildung und -Förderung zunehmend auf das zentrale Ziel der Europäischen Wirtschaftsgemeinschaft (EWG) ausgerichtet: Diese sollte ab der Wende zum 21. Jahrhundert nicht mehr nur (Land-)Technik exportieren, sondern auf dem Weltmarkt auch mit sogenannten veredelten, $d$. h. weiterverarbeiteten tierischen Produkten konkurrieren. Dafür sollten vorrangig die Böden Nordund Südamerikas als Produktionsfläche von Futtermitteln für die EWG dienen. Insbesondere mit dem Blairhouse-Abkommen von 1992 zeigte sich das entsprechende Interesse der Futtermittel produzierenden Konzerne und Länder an diesem Deal: Das Abkommen verpflichtete die EWG darauf, den eigenen Anbau proteinreicher Ölsaaten - insbesondere der Leguminose Soja - extrem zu begrenzen (Anita Idel/Tobias Reichert 2013, 143).

Offiziell stand die Politik von EWG und BRD noch bis in die 1980erJahre vorrangig unter dem Motto, den Selbstversorgungsgrad mit tierischen Produkten zu erhöhen. Damals wie heute wird meistens nicht wahrgenommen, dass für Politik und Industrie das Erreichen der Selbstversorgung für ein bestimmtes Segment immer nur ein Meilenstein auf dem Weg zum Weltmarkt war. Tatsächlich zielten ihre Maßnahmen auf die dazu notwendigen Überschüsse: mehr Milch, mehr Fleisch, mehr Eier ... Fälschlicherweise werden diese Überschüsse weiterhin häufig als vermeidbare Kollateralschäden wahrgenommen. Aber ebenso wie die Folgen für Ökologie, Tierschutz und Sozioökonomie in der Landwirtschaft und den weiterverarbeitenden handwerklichen Betrieben sind sie systemimmanent. 


\subsection{Wachsen oder Weichen am Beispiel Milchquote}

In keinem Bereich der EWG führte die (supra-)national gelenkte Politik des Wachsens und Weichens so schnell zu den gewünschten Überschüssen wie bei der Milchproduktion. Dass Milchseen und Butterberge keine Zufälle waren und auch nicht überraschend entstanden, belegen die Kühlhäuser und Kühltransporter, die immer schon bereitstanden, bevor die Über-Mengen anfielen. Dennoch ging der Plan schneller auf als gewollt, denn ein Weltmarkt, der die Überschüsse aufgenommen hätte, existierte noch nicht. Deshalb konnten die Produzenten ihre überschüssige Milch in die Intervention liefern: Sie wurde ihnen zu Marktpreisen abgekauft, ohne sie mit den Lagerkosten zu belasten.

Solange es noch nicht gelang, diese Über-Mengen am Weltmarkt abzusetzen, kam die Intervention Staat/EWG durch Lagerung und steigende Energiekosten teuer zu stehen. Die Milchquote wurde Anfang der 1980erJahre aber nicht nur wegen der hohen Kosten für die Intervention eingeführt. Hinzu kam das erhebliche Interesse der Molkereien am Wachsen einzelner Betriebe zum Zweck der Kostensenkung. Denn durch das zwangsläufig damit verbundene Weichen anderer Betriebe verringerte sich der Aufwand für die Abholung der Milch erheblich - ergänzt durch immer größere Milchtanks für immer längere Intervalle.

Wiederum war es dann kein Zufall, sondern Plan, dass die letztlich innerhalb der EWG verteilte Milchquote deutlich über dem Eigenverbrauch lag. Angetreten vorgeblich, um Überschüsse zu begrenzen, wirkte die Milchquote durch Überversorgung des Marktes nach innen: Sie diente dem Strukturwandel durch Wachsen und Weichen, da sie Großbetriebe bevorzugte. Wer seinen Kuhbestand erhöhte und den Neu- oder Ausbau von Ställen plante, erhielt per Härtefallregelung höhere Quoten: Eine krasse Benachteiligung kleiner Betriebe, die die Quotenkürzungen hinnehmen mussten und vielfach zum Aufgeben gezwungen waren.

Seit Einführung der Milchquote in der EWG wurde die weltweite Milchproduktion von 482 Millionen Tonnen um zwei Drittel auf rund 800 Millionen Tonnen erhöht. Die Länder mit der größten Überschussproduktion waren Neuseeland, USA, Australien sowie - trotz Milchquote: Frankreich, Irland und Deutschland. Seitdem wurde die in Deutschland erzeugte Milchmenge von 25,6 Millionen Liter (1985) über 28,6 (1995), 30,3 (2011), 30,6 (2014), 32,8 (2016) auf ca. 33,00 (2019) gesteigert (Milchindustrie-Verband 2019).

Die durchschnittliche Milchleistung pro Tier und Jahr wurde in Deutschland seit Ende der 1960er-Jahre mit 3.500 Litern bis 2014 mit über 8.000 Litern züchterisch mehr als verdoppelt. Gleichzeitig schrumpfte die Politik 
des Wachsens oder Weichens die Zahl der Betriebe und Milchkühe: In Deutschland melken im Jahr 1984 noch 370.000 Milchviehbetriebe und 30 Jahre später nur noch gut 75.000. Während die Gesamtzahl der Milchkühe von 5,2 Millionen (1995) auf 4,3 Millionen (2014) sank, stieg sie pro Betrieb erheblich. Und wiederum war es der Plan, dass vom Ende der Milchquote am 1. April 2015 weiterhin die Wachstumsbetriebe profitieren sollten. Aber obwohl sie die meisten Subventionen erhalten, bedrohen die geringen Auszahlungspreise für die Milch auch ihre Existenz (Katrin Jürgens et al. 2016).

\subsection{Weniger Kühe - mehr Milch}

Spezialisierung und Intensivierung nehmen weiter zu. Beispiel NordrheinWestfalen (NRW): Innerhalb von 20 Jahren - von 1991 bis 2011 - wurde dort der Bestand an Milchkühen mehr als halbiert, aber gleichzeitig stieg die gemolkene Milchmenge an (Ludger Wilstacke 2011). D. h., abgeschafft wurden Weidetiere, die Milch wesentlich aus Gras und deshalb viel weniger in Nahrungskonkurrenz zum Menschen bilden. Gleichzeitig wurden die Lebensbedingungen der verbleibenden Hochleistungskühe umso intensiver: Krankheitsanfälligkeit und Stress sowie Berufskrankheiten wie schmerzhafte Entzündungen des Euters und der Klauen (Holger Martens 2021) sind der Preis, den einseitig auf Hochleistung selektierte Kühe zahlen. Untrennbar damit verbunden klafft die Schere zwischen Milchhöchstleistung und Fleischansatzvermögen, so dass männliche Kälber an Wert verlieren und tendenziell vernachlässigt werden (Tanja Busse 2015; Darko Janjevic 2019).

Heute stammen laut Welternährungsorganisation FAO mehr als Zweidrittel der in der Landwirtschaft der EU verfütterten Proteine aus Importen: Ganz entgegen ihrer eigenen Verlautbarungen ernährt die EU mit dem Export ihrer gewollten Überschüsse nicht die Welt, sondern produziert diese auf Kosten anderer (Anita Idel/Andrea Beste ${ }^{32019) . ~ I n ~ W a h r-~}$ heit hängt die EU am Tropf. 


\section{Billig ist nur scheinbar billig}

\subsection{Zur Externalisierung von Kosten}

Die mit der Industrialisierung und Maximierung verbundenen Kosten für die Umwelt (die nicht tierische Mitwelt) werden durch die Preise im Regal nicht abgebildet, aber in der Öffentlichkeit neben den sozialen Problemen und denen des Tierschutzes immer wahrnehmbarer:

- der Verlust von Lebensräumen - blühenden Weiden, Wiesen und Hecken - und ihren pflanzlichen und tierischen Bewohnern, darunter insbesondere den Insekten,

- die Zunahme von Resistenzen bei Antibiotika - gefolgt von Antiparasitika, Insektiziden, Herbiziden, Pestiziden und Desinfektionsmitteln,

- der globale Anstieg der Temperaturen mit Zunahme extremer Wetterereignisse,

- die Belastung von Gewässern und Böden mit ökologisch und gesundheitlich problematischen Rückständen,

- der Verlust und die Belastung

- von Grundwasser und anderen Gewässern,

- von fruchtbaren Böden.

\subsection{Kein Unfall, sondern systemisch bedingt}

Die generelle Schlussfolgerung aus dem ersten UN-Weltagrarbericht (Beverly McIntyre et al. [Hg.] 2009) lautet: „Weiter wie bisher ist keine Option" (Maria Heubuch [Hg.] 2019). Es handelt sich nicht um Kollateralschäden einer ansonsten verantwortbaren Entwicklung, sondern um das System selbst: ein krankes Agrar- und Ernährungssystem - zunehmend chronisch und partiell immer auch akut krank. Statt mit Pestiziden, Medikamenten und synthetischem Stickstoffdünger weiter das kranke Agrarsystem zu stützen, benötigen Tier, Umwelt und Mensch eine (Agrar-)Politik, die durch Forschung, Ausbildung und finanzielle Förderung die Voraussetzungen für nachhaltige Entwicklung schafft. Ebenfalls bereits 2009 belegte eine Studie (Johan Rockström et al. 2009) das Ausmaß der überreizten neun planetaren Grenzen: allen voran der Verlust der biologischen Vielfalt, die direkt mit der Überlastung der Biosphäre durch Stickstoff verbunden ist, sowie dem Klimawandel.

Die zwangsläufigen Folgekosten werden externalisiert - mit Auswirkungen auf Tiere, Natur, Umwelt und Klima ebenso, wie auf die sozialen Be- 
dingungen der Menschen in den Betrieben. Das bedeutet für Bäuerinnen und Bauern weltweit: Sie werden immer abhängiger von der Chemischen, der Agrar- und der Lebensmittelindustrie, die ihrerseits durch horizontale und vertikale Integration immer mächtiger wird. Innerhalb der Landwirtschaft profitieren große Betriebe, die die Economies of Scale nutzen können. In diesem Sinne heißen Überschüsse nun Exportprodukte. Z. B. liegt der Anteil europäischer Hersteller am Milchmarkt in Burkina Faso inzwischen bei fast 90 Prozent (Jürgen Maier 2017).

\subsection{Weiterhin Fokus auf Intensivierung, Überschüsse und Exportorientierung ...}

Laut EU-Kommission werden staatliche Subventionen gezielt dazu genutzt, die Betriebe wettbewerbsfähig für den Weltmarkt zu machen. Jährlich stehen 55 Milliarden Euro an Direktzahlungen für die EU-Landwirtschaft zur Verfügung. Laut Zentrum für Europäische Wirtschaftsforschung (ZEW) erhalten die 80 Prozent der Betriebe mit geringem Einkommen nur 25 Prozent der Direktzahlungen, die zehn Prozent mit dem höchsten Einkommen dagegen 55 Prozent. Gleichzeitig steigen die betriebswirtschaftlichen Risiken. EU-weit haben fast 20 Prozent der Bauernhöfe im Zeitraum von 2003 bis 2010 aufgegeben (Reinhild Benning/Tobias Reichert 2016). Generell und insbesondere im Bereich der Milch produzierenden Betriebe verschärfen die geringen Erzeugerpreise diese Tendenz.

Die Konzerne treiben die Industrialisierung entlang der gesamten Wertschöpfungskette voran - von Acker und Tierstall bis zu Regal und Theke. Während sich auf der Ebene der landwirtschaftlichen Betriebe die Entscheidung als Wachsen oder Weichen darstellt, lautet das politische Konzept dahinter Weichen und Wachsen: Die Zahl der Betriebe schrumpft dramatisch - zugunsten der Industrialisierung und immer größerer Betriebe. Aber mit Größenordnungen wie in Nord- und Südamerika sowie Australien kann die EU nicht nur nicht konkurrieren, sondern sollte es aufgrund der damit zwangsläufig verbundenen ökologischen, sozioökonomischen und (tier-)gesundheitlichen Belastungen und Schäden auch nicht.

Die Welternährungsorganisation (FAO) wird ihrer Verantwortung nicht gerecht - im Gegenteil: Statt wie der UN-Weltagrarbericht (Beverly McIntyre et al. [Hg.] 2009, 4) eine Internalisierung dieser Kosten zu fordern, rechnet die FAO hinsichtlich tierischer Produkte und insbesondere beim Fleischkonsum den Verbrauch von heute mit dem Bevölkerungszuwachs zur Nachfrage von morgen hoch. Damit suggeriert sie einen künftigen Bedarf und argumentiert damit für einen forcierten Ausbau industrieller Strukturen (Anne Mottet/Giuseppe Tempio 2017). Stattdessen läge es 
in der Verantwortung der FAO, angesichts der Zerstörung von Ressourcen und der Klimakrise alles zu unternehmen, um den Trend umzukehren und nachhaltige Produktion zu unterstützen.

\section{4 ... und Freibandel}

Mit Mercosur bekommen Freihandelsabkommen insbesondere für die Rinder haltenden Fleisch produzierenden Betriebe in der EU eine neue Qualität. Am 20. Dezember 1985 entschied der Ministerrat der EWG, den Einsatz von Hormonen in der Tiermast zu verbieten. Noch nie war seitdem die Gefahr sinkender Standards zulasten der europäischen Betriebe mit Weidehaltung so groß wie jetzt. Zwar gibt es für Drittländer neben der Quote für hormonfrei auf der Weide erzeugtes Rindfleisch seit Oktober 2014 bereits eine erweiterte Quote für hormonfrei in Intensivmast erzeugtes Rindfleisch. Aber mit Mercosur geht es darüber hinaus vorrangig darum, den Export von Autos etc. aus der EU zu befördern und dafür zum partiellen Ausgleich der Handelsbilanzen Abstriche bei den Standards von Agrarimporten zu akzeptieren (Ismail Doga Karatepe et al. 2020; GRAIN 2019; Martin Häusling 2018; Anita Idel 2018).

\subsection{Acker-versus Grasland}

Da viel weniger in als an der Landwirtschaft verdient wird, profitieren von der Umwandlung von Gras- in Ackerland weniger die landwirtschaftlichen Betriebe selbst als vorrangig diejenigen Unternehmen, die landwirtschaftliche Betriebe beliefern, und diejenigen, die deren Produkte weiterverarbeiten: $\mathrm{Zu}$ den Zulieferern zählen neben der Chemischen Industrie - mit Saatgut, Mineral- und synthetischem Stickstoffdünger, Pestiziden sowie mit Futtermitteln, Antibiotika, Antiparasitika, Hormonen etc. - die Landmaschinenindustrie, die Stallbau- und -einrichtungsfirmen und die Tierzuchtunternehmen. Neben den Transportunternehmen dominieren als Abnehmer im tierischen Bereich die Molkerei-, Schlacht- und Lebensmittelkonzerne. Entsprechend konzentriert sich das Interesse der Industrie auch bei der Herstellung tierischer Produkte auf den Ackerbau. Denn an den mit dem Anbau von Kraftfutter verbundenen Inputs lässt sich für die Industrie viel mehr verdienen als am Grasland. Der (ökologische und) Klima-Fußabdruck der Tierproduktion nimmt mit der weltweiten Expansion der Milch- und Fleisch-Konzerne dramatisch zu (GRAIN/IATP 2018). 
3. Nicht angemessenes Studiendesign - Was wird untersucht? Was wird nicht untersucht? (1)

Ob Futter oder Dünger: Die Kosten dieser Inputs lohnen sich nur, wenn die Tiere und ebenso die Pflanzen die darin enthaltenen Nährstoffe maximal verwerten können. Deshalb sind die Zuchtziele auf Maximierung ausgerichtet - durch Spezialisierung und Intensivierung: bei den Tieren auf mehr Milch oder mehr Fleisch oder mehr Eier - und das in immer kürzerer Zeit. Grundsätzlich können Forschungsergebnisse durch das jeweilige Forschungsdesign entscheidend beeinflusst werden. Insbesondere hinsichtlich der Bewertung der Produktivität und der Umweltrelevanz intensiver Agrarsysteme einerseits und nachhaltiger andererseits wirkt der jeweils ausgewählte Zeitraum als wesentlicher Einflussfaktor. Deshalb muss, wer Nachbaltigkeit wirklich ernst nimmt, somit immer auch die Frage untersuchen, ob die untersuchten Agrarsysteme und insbesondere die vermeintlich produktiveren auf Dauer geeignet sind, die lebenswichtigen Ressourcen - Bodenfruchtbarkeit, Gewässerqualität, biologische Vielfalt - zu erhalten (Anita Idel/Andrea Beste ${ }^{32019) .}$

\subsection{Kurzer versus längerer Zeitraum: ökologischer und klimatischer Fußabdruck}

Die meisten Studien sind auf (zu) kurze Untersuchungszeiträume beschränkt, während die negativen Folgen industrialisierter Agrarsysteme der ökologische und klimatische Fußabdruck - mit der Zeit kumulieren. Deshalb kommen Studien mit den üblichen kurzen Untersuchungszeiträumen von nur einer Vegetationsperiode (wenn beispielsweise die Erntemenge einer bestimmten Pflanze auf einem konventionellen Acker mit der auf einem ökologischen verglichen wird) meistens zu dem Ergebnis, Agrarsysteme mit höherem Industrialisierungsgrad seien diejenigen mit dem geringeren Fußabdruck - und somit besser.

Ähnlich unwissenschaftlich ist es, den ökologischen und klimatischen Fußabdruck von Kühen mit unterschiedlicher Jahresmilchleistung (wenn beispielsweise zwei 5.000-Liter-Kühe mit einer 10.000-Liter-Kuh verglichen werden) zu bewerten, wenn die Datenbasis auf den Zeitraum nur einer Laktation beschränkt ist. Hochleistungskühe haben vergleichsweise kurze Nutzungsdauern. Im Durchschnitt liegen diese bereits seit Jahrzehnten bei weniger als drei Jahren. Die Länge des Lebenszeitraums, währenddessen die abgehende und die sie ersetzende Kuh parallel leben, d. h. fressen, Exkremente hinterlassen und rülpsen, beeinflusst die Ergebnisse wesentlich. Dieser gemeinsame Lebenszeitraum ist anteilig umso größer, je kürzer die 
Nutzungsdauer einer Kuh ist, und muss mitberücksichtigt werden (Anita Idel/Andrea Beste 32019, 47).

\subsection{Vermeintliche Produktivität}

Immer, wenn einseitig auf eine Leistung selektiert wird, die nur das weibliche Tier erbringen kann - Milch oder Eier -, führt das zwangsläufig zu einem nur geringen Fleischansatzvermögen.

So bedingt das einseitige Zuchtziel Milchleistung bei Kühen die nur geringe Masttauglichkeit der Brüder und Söhne; denn die hohen Milchleistungen resultieren aus einem veränderten Stoffwechsel: Die durch das Futter aufgenommene Energie wird vorrangig zum Euter transportiert. Deshalb scheiden mangels Erfolgsorgan die männlichen Tiere der Milchviehrassen einen wesentlichen Anteil der aufgenommenen Nahrungsenergie mit den Exkrementen aus. So ist der züchterische Erfolg - die Produktivität der weiblichen - untrennbar mit der mangelnden Rentabilität der männlichen Tiere verbunden (Stefan Probst et al. 2019). Deshalb ist es unwissenschaftlich, die Berechnung des Fußabdruckes auf die hohe Milchleistung zu beschränken und dabei die geringe Fleischbildung auszuklammern.

Damit bei der Produktion von Milch erst gar keine männlichen Kälber entstehen, wird seit den 1970er-Jahren in die Forschung für das sogenannte Sexen von Rindersperma investiert. So sollen für die künstliche Befruchtung der weiblichen Tiere Spermien mit Geschlechtschromosomen für männliche Tiere identifiziert werden, um sie zu eliminieren (Primoz Klinc/Detlef Rath 2005).

Aber es ist für beide Geschlechter keine ethisch verantwortbare Lösung, wenn Züchtung darauf zielt,

- die Milchleistung einseitig zu steigern, was Qualzucht für die weiblichen Tiere bedeutet, und

- das männliche Geschlecht komplett zu eliminieren.

Entsprechend führt auch die einseitige Selektion von Hühnern auf die Legeleistung der Hennen zwangsläufig zu einem nur geringen Fleischansatzvermögen (Anita Idel 2020). ${ }^{1}$

1 Zum Problem des Tötens männlicher Küken sowie der Auswirkungen der Leistungszucht beim Geflügel auf das Tierwohl siehe Anita Idel 2007 und 2011; Bernhard Hörning 2017; Bundesverwaltungsgericht 2019; Merlind Theile 2019. Auf die 


\subsection{Kurzer versus längerer Zeitraum und vermeintliche Produktivität}

Die Notwendigkeit, längere Zeiträume zu untersuchen, zeigt sich auch bei Systemvergleichen zwischen dem biologischen und dem konventionellen Anbau bezüglich der Biomassebildung bzw. der Erntemengen. Die meisten dieser Studien werden auf nur eine Wachstumsperiode beschränkt. Aber länger- und langfristig wirken sich beide Systeme völlig unterschiedlich auf die Quantität und Qualität der verfügbaren Ressourcen und damit auf die reale künftige Produktivität aus (Jürn Sanders/Jürgen Hess [Hg.] 2019). Deshalb ist es unwissenschaftlich, aus nur einer Vegetationsperiode eine Schlussfolgerung hinsichtlich der Produktivität zu ziehen.

Der Terminus von den Grenzen des Wachstums bestimmt seit 1972 die Kritik an der weltweiten Ressourcenzerstörung und deren Zusammenhang mit dem Verbrennen fossiler Energie (Donella H. Meadows et al. 1972). Angesichts des Artensterbens, der Klimakrise und der Bodenerosion konkretisierte der UN-Welt-Agrarbericht (2004-2009) diese desaströse Entwicklung für die Landwirtschaft einerseits und die Potenziale der Agrarökologie andererseits. Eine Leitfrage lautete, ob der andauernde Welthunger und der eskalierende Ressourcenverlust trotz oder wegen des Mainstreams in der agrarwissenschaftlichen Forschung, Lehre und Praxis erfolgt. Der Bericht kommt nach vier Jahren zu dem Schluss, dass in Forschung und Lehre eine hochriskante Entkopplung stattfindet: Die Industrialisierung und die durch sie verursachten Wirkungen werden viel zu wenig in einen Zusammenhang gebracht. Verstärkt wird dieses Problem dadurch, dass in der Folge die Externalisierung der Kosten dazu beiträgt, die Schäden nicht ausreichend wahrzunehmen. Salopp formuliert: Billig ist nur scheinbar billig (Beverly McIntyre et al. [Hg.] 2009). Ebenfalls 2009 veröffentlichte der schwedische Resilienzforscher Johan Rockström die mit einem großen Team erarbeiteten Ergebnisse zu den planetaren Grenzen. Demnach besteht - über die Zerstörung von Lebensraum und den Einsatz von Chemie hinaus - ein positiver Zusammenhang von dramatischem Ausmaß zwischen dem immer höheren Einsatz von Stickstoff und dem Artensterben (Johan Rockström et al. 2009). Über fast drei Jahrzehnte dokumentierten Forscher in Deutschland den Verlust der biologischen Vielfalt in Naturschutzgebieten: Der Rückgang der Insektenbiomasse beträgt mehr als 75 Prozent (Caspar A. Hallmann et al. 2017). Wie sehr der Biodiversitätsverlust auch welt-

gravierende Problematik, dass systematische Verletzungen des Tierschutzrechts in der Massentierhaltung strafrechtlich kaum geahndet werden, kann an dieser Stelle nur hingewiesen werden (Jens Bülte 2018). 
weit in den vergangenen zehn Jahren immer weiter zugenommen hat, verdeutlicht der UN-Biodiversitätsbericht (IPBES 2019). Der Direktor des IPBES, Sir Robert Watson, ist Chemiker und Meteorologe und war u. a. von 1997 bis 2002 Direktor des UN-Weltklimarates (Intergovernmental Panel on Climate Change IPCC) und von 2003 bis 2009 Direktor des UN-Weltagrarberichtes International Assessment of Agricultural Knowledge, Science and Technology for Development (IAASTD). Kaum bekannt ist, wie dramatisch sich der Rückgang der Beweidung auf die mit der Dungfauna verbundenen Nahrungsketten auswirkt: Eine Kuh bildet über $10 \mathrm{t}$ Dung pro Jahr und bietet damit Futter für über $100 \mathrm{~kg}$ Insektenbiomasse (Jörn Buse 2020).

\section{Nicht angemessenes Studiendesign - Was wird untersucht? Was wird nicht untersucht? (2)}

\subsection{Mythos: Rinder sind schlechte Futterverwerter}

Warum kommen so viele Studien zu dem Schluss, Rinder seien schlechte Futterverwerter? Seit den 1970er-Jahren zielt die züchterische Selektion bei allen landwirtschaftlich genutzten Tierarten auf kurzfristige Hochleistung. Beim Rind bedeutet dies je nach Rasse: viel Milch oder viel Fleisch - und das in möglichst kurzer Zeit. Obwohl sie geniale Grasverwerter sind, wurde schon damals an Universitäten gelehrt, Rinder seien schlechte Futterverwerter (Pierre Gerber et al. 2013). Das beruht damals wie heute auf Studien, die aufgrund des Studiendesigns zwangsläufig zu dieser Schlussfolgerung führen. Denn die Rinder werden dabei nicht in einem für Wiederkäuer artgerechten System und somit nicht an dem gemessen, was sie besonders gut können: vorrangig Gras verdauen.

Stattdessen wird ihre Ernährung intensiviert mit proteinreichem Kraftfutter aus energieaufwändigem Ackerbau. Nur deshalb generiert die Intensivfütterung die absurde Schlussfolgerung, Rinder seien im Vergleich zu Huhn und Schwein schlechte Futterverwerter. Dabei handelt es sich letztlich um eine unwissenschaftliche Schlussfolgerung. Denn dass die Verdauungstrakte von Menschen (Alles-Esser) sowie die von Schweinen und Hühnern (Alles-Fresser) kalorienreiche Lebensmittel bzw. konzentriertes Futter wesentlich besser verwerten können als die Verdauungssysteme von Wiederkäuern und anderen Grasfressern, ist bekannt, bevor diese Studien beginnen (Anita Idel ${ }^{82021) .}$ 


\subsection{Mythos: Rinder sind Klima-Killer}

Warum kommen so viele Studien zu dem Schluss, Rinder seien Klima-Killer? Auch bei diesen Untersuchungen bedingt das Studiendesign diese fast zwangsläufige Schlussfolgerung (Anita Idel ${ }^{82021}$ ).

\subsubsection{Studiendesign: beschränkt auf Emissionen}

Denn für die meisten dieser Studien gilt:

- Erstens sind sie auf die Messung von Emissionen beschränkt und

- zweitens vergleichen sie den Wiederkäuer Rind mit den Allesfressern Schwein und Huhn (und manchmal auch mit Fischen und Menschen), ohne zwischen einem Low-Input-System einerseits und einem energieund ressourcenintensiven System andererseits zu unterscheiden.

Das gilt auch für den weitaus überwiegenden Teil der von der FAO veröffentlichten Studien zum Beitrag der Tierhaltung zur Klimakrise. „Cattle are the main contributor to the sector's emissions with about 4.6 gigatonnes $\mathrm{CO}_{2}$-eq, representing 65 percent of sector emissions" (Pierre Gerber et al. 2013, 15). Ignoriert wird dabei das Potenzial zum Humusaufbau und der damit verbundenen Kohlenstoffspeicherung, das bei nachhaltigem Weidemanagement am größten ist (FAO 2010; Richard Conant 2010).

\subsubsection{Studiendesign: beschränkt auf Methan-Emissionen}

Ein großer Teil der Studien setzt die Systemgrenzen sogar noch enger. So müssen insbesondere jene Studien wissenschaftskritisch hinterfragt werden, in denen seit Jahrzehnten die Erforschung der Emissionen durch die Produktion von Milch oder Fleisch auf ein einzelnes Klimagas begrenzt wird - das Methan (CH4) (Julie Wolf et al. 2017; Dorien Van Wesemael et al. 2019).

Tatsächlich rülpsen Rinder das Gas $\mathrm{CH} 4$ und dieses ist 25-mal so relevant für das Klima wie $\mathrm{CO}_{2}$. Methan entsteht vorrangig durch Mikroorganismen im Pansen, die Nährstoffe aus dem Gras für die Rinder verfügbar machen. Hühner und Schweine scheiden nur vergleichbar geringe Mengen an Methan aus. Aber auch das ist bereits bekannt, bevor die Studien beginnen. 
Wissenschaftlich erforderlich wäre, unterschiedliche Agrarsysteme hinsichtlich ihrer gesamten Klimawirkung zu vergleichen (Anita Idel/Tobias Reichert 2013). Stattdessen wird überwiegend auf Methan beschränkt zwischen verschiedenen Tierarten verglichen.

Überwiegend ist das Design der Studien darauf ausgerichtet, die Methan-Emissionen pro Kilogramm Milch oder Fleisch zu verringern. Deshalb suggeriert das zwangsläufige Ergebnis: Grasverdauung reduzieren und mehr Kraftfutter füttern. D. h., das System mit der höheren Intensität und dem höheren Industrialisierungsgrad erscheint als das - vermeintlich - bessere (Harald von Witzke et al. ${ }^{4} 2014$ ).

Dementgegen wird die Klimarelevanz von Kraftfutter aber erst offenbar, wenn dessen Produktion und die damit verbundenen Kosten mitberechnet werden. Dazu zählen die Herstellung von chemisch-synthetischem Stickstoffdünger, die erhebliche $\mathrm{CO}_{2}$ - und Methanemissionen verursacht, sowie seine Anwendung, die zur Bildung von Lachgas $\left(\mathrm{N}_{2} \mathrm{O}\right)$ führt: $\mathrm{N}_{2} \mathrm{O}$ ist 12-mal so klimarelevant wie Methan und 300-mal so klimarelevant wie $\mathrm{CO}_{2}$ (Rona L. Thompson et al. 2019; Magdalena Necpalova et al. 2018; Mark A. Sutton et al. [Hg.] 2011). Hinzu kommen die Klimaeffekte der für den Ackerfutterbau erforderlichen desaströsen Rodungen von (Regen-)Wald und ebenso der Umbrüche von Dauergrasland. Diese sogenannten Landnutzungsänderungen (Land Use, Land-Use Change and Forestry LULUCF) setzen extreme Mengen an $\mathrm{CO}_{2}$ frei. Obwohl sie weltweit den größten Beitrag der Landwirtschaft zum Klimawandel verursachen, rechnet die EU-Administration sie nicht den landwirtschaftlichen Emissionen $\mathrm{zu}$, sondern hat für die LULUCF eine eigene Gruppe definiert (BMU 2019, 48-51).

\subsection{Mythos: Rinder haben einen besonders großen Flächen- und Wasserrucksack (Fußabdruck)}

\subsubsection{Flächenrucksack}

Warum kommen so viele Studien zu dem Schluss, dass Rinder besonders viel Land verbrauchen?

Die Tierhaltung ist weltweit der mit Abstand größte Landnutzer. Ob sich das gut oder schlecht - oder neutral - auswirkt, hängt vom jeweiligen Agrarsystem und somit vom Wie der Landnutzung ab. Wie groß die Unterschiede sein können, zeigt sich insbesondere bei der Rinderhaltung: Nachhaltiges Beweidungsmanagement fördert Bodenfruchtbarkeit, Wasserqualität, Wasserspeicherkapazität sowie biologische Vielfalt und trägt zur Kli- 
maentlastung bei (Richard Conant 2010; FAO 2010). Im Gegensatz zu genügsamen Rassen erfordert einseitige Hochleistung auf Milch oder Fleisch hohe Kraftfuttermengen. Das bedeutet: Die Intensität ihres Fütterungssystems bedingt, wie energieaufwändig und klimarelevant einerseits oder nachhaltig und artgerecht andererseits Rinder in unterschiedlichen Agrarsystemen gehalten werden. Wenn aber nicht Systeme - gras- oder kraftfutterbasiert - miteinander verglichen werden, sondern der Wiederkäuer Rind mit den Allesfressern Schwein und Huhn, liegt auch diesen Studien ein wissenschaftlich nicht fachgerechter und aufgrund der suggerierten Schlussfolgerungen kontraproduktiver Ansatz zugrunde. Das zeigt auch eine Studie über die USA zur Flächeneffizienz aus dem Jahr 2014:

„Beef production requires 28 -times more land [...] than the average of the other livestock categories. [...] The study thus elucidates the multiple environmental benefits of potential, easy to implement dietary changes, and highlights the uniquely high resource demands of beef. [...] Preliminary analysis of three staple plant foods shows two- to sixfold lower land, GHG, and $\mathrm{Nr}$ [reactive nitrogen] requirements than those of the non-beef animal derived Calories" (Gidon Eshela et al. 2014, 1).

Auch hier wird nicht nach dem Wie der Nutzung differenziert; somit ist es unwissenschaftlich,

- die Nutzung von Böden generell mit ihrem Verbrauch gleichzusetzen;

- bei den Berechnungen nicht zwischen Dauergrasland und Ackerland zu differenzieren; denn dann schneiden aufgrund ihres kleineren Flächenrucksacks

- Tierarten, die mehr Futter vom Ackerland erhalten als der Wiederkäuer Rind, vergleichsweise besser ab,

- Fütterungssysteme für den Wiederkäuer Rind umso besser ab, je mehr Futter er vom Ackerland erhält.

- Systeme immer umso schlechter ab, je mehr mit Grasland gewirtschaftet wird.

\subsubsection{Wasserrucksack}

Warum kommen so viele Studien zu dem Schluss, dass Rinder besonders viel Wasser verbrauchen?

Der Mythos, das Rind sei der größte Wasserverbraucher unter den landwirtschaftlich genutzten Tieren, folgt einer ähnlichen Logik wie der, wo- 
nach Rinder die größten Landverbraucher sind. Für die Erzeugung eines Kilogramms Rindfleisch werden Zahlen bis 100.000 Liter Wasser gehandelt (David Pimentel/Marcia Pimentel 2003; David Pimentel et al. 1997). Solchen Berechnungen liegt nicht nur die künstliche Bewässerung zugrunde, sondern auch der Regen, der auf das Land fällt, von dem das Futter stammt. Deshalb schneidet wiederum zwangsläufig die industrialisierte Produktion, da sie ja einen vermeintlich besseren, weil geringeren Flächenbedarf hat, besser ab und auf Grasland basierende Fütterungssysteme entsprechend schlechter.

Dass die nachhaltige Nutzung von Dauergrasland mit Rindern auch beim Wasser mit keiner anderen Nutzung konkurriert und somit gerade nichts auf Kosten der menschlichen Ernährung verbraucht wird, gerät dabei völlig aus dem Blick. Tatsächlich kommt Grasland eine unverzichtbare Bedeutung für die Regeneration des Grundwassers zu. Erstens quantitativ: Grasland ist das größte Biom; weltweit bedeckt keine Pflanzengesellschaft mehr Erdoberfläche. Zweitens qualitativ: Ackerland wird Jahr für Jahr mit viel mehr Mineraldüngern, Herbiziden, Pestiziden, Antibiotika etc. belastet (Christopher J. Topping et al. 2020).

\section{Die Ko-Evolution von Gräsern, Grasland und Weidetieren}

\subsection{Größtes Biom, größte Perma- und größte Mischkultur}

Um die Potenziale nachhaltiger Beweidung wahrnehmen, nutzen und bewerten zu können, ist in der Forschung ein ökosystemarer - holistischer Ansatz in Raum und Zeit erforderlich. Das betrifft neben den langfristigen Auswirkungen auf das Klima vor allem diejenigen auf die Basisressourcen - die biologische Vielfalt, die Bodenfruchtbarkeit und die Gewässerqualität. Aber Grasland spielt in der Ausrichtung der EU-Agrarpolitik und -forschung eine sehr untergeordnete Rolle.

Grasland nimmt mehr als 30 Prozent der Landfläche des Planeten ein. Trotz dramatischen Umbruchs ist es als größtes irdisches Biom zudem auch immer noch die größte Perma- sowie Mischkultur. Bezogen auf die weltweit landwirtschaftlich genutzte Fläche (LN) sind - wie in der Schweiz - 70 Prozent Grasland. In der EU entfallen noch circa 40 Prozent der LN auf das Grasland, darunter Österreich mit 54 und Deutschland mit 28 Prozent (Wolfgang Willner 2013; Umweltbundesamt 2020).

Die (Klima-)Forschung konzentriert sich überwiegend auf das Ackerland. Deshalb bleiben die speziellen Wachstumsdynamiken des Graslandes und die Potenziale nachhaltiger Beweidung für Bodenfruchtbarkeit, Ge- 
wässerqualität und Artenvielfalt und in der Folge für das Weltklima und die Welternährung vielfach unerkannt und weitgehend ungenutzt.

\subsection{Die Steppengenese der Kornkammern}

Verantwortbare Agrar- und Ernährungspolitik müsste grundsätzliche Fragen zur Basisressource menschlicher Entwicklung untersuchen: Wie ist Bodenfruchtbarkeit entstanden, bevor sich Menschen sesshaft machten und $\mathrm{zu}$ ackern und zu gärtnern begannen? Und wo liegen heute die fruchtbarsten Großebenen der Welt? In sogenannten Kornkammern werden seit Jahrhunderten Getreide, Mais und Soja in riesigen Monokulturen angebaut. Dazu zählen die Schwarzerdeböden (Tschernoseme) der Prärien in Nordamerika, der Ukraine, der Puszta in Ungarn, des Bărăgans in Rumänien, des Pannonischen Tief- und Hügellandes in Österreich, der Böden in den deutschen Tieflandsbuchten sowie Kasachstans, der Mongolei und Chinas (Mandschurei) und auch der subtropischen Pampas in Argentinien und Uruguay. Das gemeinsame Vielfache: Sie alle haben eine Genese als Steppenboden - und das bedeutet Beweidung (Anita Idel ${ }^{82021 \text { ). }}$

Ihre hohen Lössanteile boten eine günstige Voraussetzung für die Entwicklung von Bodenfruchtbarkeit. Belebt wurden sie wie alle Böden von oben: Jahrtausende lange Beweidung förderte das ober- und unterirdische Pflanzenwachstum. Durch die Wurzelexsudate und die Verrottung der Wurzelbiomasse entstanden im Mittleren Westen sogar bis zu sechs und in der Ukraine bis zu drei Meter dicke Humusschichten (organische Bodensubstanz) (Turi Fileccia et al. 2014).

\subsection{Dauergrasland ist anders: Gräser brauchen den Biss}

Gräser brauchen den Biss. Kein Dauergrasland bleibt flächendeckend erhalten, wenn es dauerhaft ungenutzt bleibt. Und Nutzung bedeutete über 59,999 Millionen Jahre: Beweidung (Jörg Pfadenhauer/Frank Klötzli 2014, 13, $155 \mathrm{ff})$. Warum löst Beweidung von Gräsern einen Wachstumsimpuls aus, während Baumschösslinge durch den sogenannten Verbiss eingehen oder zumindest eine Wachstumsdepression erfahren? Die Gründe für diese völlig entgegengesetzten Effekte der Beweidung liegen im fundamentalen Unterschied hinsichtlich ihrer Wachstumsdynamik: Bäume und andere Pflanzen wachsen aus dem Spross/den Sprossen heraus. Viele wehren sich mit hohem energetischem Aufwand gegen den Verbiss durch pflanzenfres- 
sende Tiere: Sie bilden Bitterstoffe, Toxine, Dornen oder Stacheln. Gräser wachsen von unten nach - aus der Basis. Das ist das Ergebnis ihrer Anpassung, sodass sie von der Beweidung profitieren.

Dauergrasland übersteht als größte Mischkultur vier Extreme - Hitze und Kälte sowie Trockenheit und Nässe: Gräser wachsen bei extremer Kälte oberhalb der Baumgrenzen ebenso wie in der Hitze der Kalahari. Gräser leben in feuchten Gebieten wie den Auenlandschaften ebenso wie im meist trockenen Sahel. Das Erfolgsprinzip des Graslandes liegt in seiner Biodiversität. Die Vielfalt dieser verschiedenen regional und lokal durch natürliche Selektion angepassten Gräser findet sich auch als Samen im Boden. So können einzelne Gräser dieser Mischkulturen in kürzester Zeit keimen und wachsen - und so auf die jeweiligen Umweltbedingungen extrem flexibel reagieren. Das gilt auch für alle Übergangsformen zwischen nass und kalt sowie trocken und heiß. So überleben Grasgesellschaften auch in Regionen, in denen sich Extreme - Überschwemmungen und Trockenzeiten - abwechseln. Solche Systeme bezeichnet man heute als resilient. Aber die Samenbank im Boden gerät infolge des Pestizideinsatzes und der Überdüngung dramatisch unter Druck.

\subsection{Kohlenstoff weltweit in Grasland- und Waldökosystemen}

Während generell das Rind und speziell das Weiderind in Wissenschaft, Politik und Öffentlichkeit als Klima-Killer Nr. 1 gilt, wird im Rahmen biologischer Antworten auf die Klimakrise nur der Wald als Klima-Retter wahrgenommen. Dabei wird völlig ignoriert, dass Dauergrasland völlig anderen Wachstumsdynamiken folgt als Wald: Bäume bilden überwiegend oberirdische Biomasse, das Holz, während Gräser infolge ihrer 60 Millionen Jahre währenden Ko-Evolution mit den Weidetieren vorrangig über die Exsudate und die Verrottung ihrer Wurzeln zur Bildung von Bodenbiomasse beitragen. In der Folge ist in den Böden unter dem Grasland weltweit erheblich mehr Kohlenstoff gespeichert als in Waldböden (Richard Conant 2010, 22).

\subsubsection{Vergleiche innerhalb eines bestimmten Zeitraumes}

Grasland kann je nach Temperatur, Sonnenexposition und Niederschlag in jeder Jahreszeit wachsen. Auf der Nordhalbkugel wirkt vorrangig Kälte, auf der Südhalbkugel vorrangig Trockenheit als begrenzender Faktor für 
das Graswachstum. Für Bäume beginnt die Vegetationsperiode im Frühjahr; mit dem Vergilben der Blätter endet das Wachstum.

Das Stamm- und Astholz stellt das Produkt Jahrzehnte oder Jahrhunderte langer Speicherung der Bäume dar. Für Vergleiche zwischen Wäldern und Grasland hinsichtlich der Speicherung des in der (allein oberirdischen) pflanzlichen Biomasse gebundenen organischen Kohlenstoffs ist es deshalb wissenschaftlich erforderlich, den Faktor Zeit zu berücksichtigen: Dazu muss die während dieses Zeitraumes gewachsene Grasbiomasse visualisiert bzw. gemessen werden.

\subsubsection{Vergleiche zu einem bestimmten Zeitpunkt}

Will man den gesamten Gehalt an organischem Kohlenstoff von einem Hektar Wald und einem Hektar Grasland zu einem bestimmten Zeitpunkt vergleichen, dann muss nicht nur die pflanzliche Biomasse, sondern die gesamte organische Biomasse berechnet werden. Denn der organische Kohlenstoff des Graslandes ist hauptsächlich in der organischen Bodensubstanz (OBS) gespeichert: 50 Prozent mehr als in Waldböden (Pawlok Dass et al. 2018; Richard Conant 2010, 22; FAO 2010).

\subsection{Entscheidende Unterschiede von Grasland- und Waldökosystemen}

\subsubsection{Wurzel-Spross-Verhältnis}

$\mathrm{Zu}$ den wichtigsten Unterschieden hinsichtlich der Kohlenstoffspeicherung gehört ein quasi unsichtbarer: Grasland verfügt über besonders viel Wurzelmasse im Verhältnis zum oberirdischen Bewuchs. Das WurzelSpross-Verhältnis (Root-Shoot-Ratio) liegt bei Gräsern zwischen 2:1 und 20:1 zugunsten der Wurzelmasse (Bäume 1:2) (Jörg Pfadenhauer/ Frank Klötzli 2014, 156, 426). In der Folge stammt bei Grasland der meiste im Boden gespeicherte organische Kohlenstoff direkt aus den Graswurzeln: Er ist überwiegend root-derived und weniger aus oberirdisch verrotteter Pflanzenbiomasse entstanden (Christopher Poeplau 2016). 


\subsubsection{Wasserspeichervermögen}

Pflanzen mit einem höheren Anteil an Fein-Wurzeln können sowohl effektiver Nährstoffe im Boden aufnehmen als auch erheblich mehr Kohlenstoff im Boden speichern. Populär ausgedrückt, sind die Wurzeln von heute der Humus von morgen (Anita Idel ${ }^{8} 2021$ ).

Ein weiterer wichtiger Unterschied liegt in der hohen Wasserspeicherkapazität und dem Potenzial, Wassererosion zu verringern (Gwendolyn Gyssels et al. 2005). Dauergrasland bietet die sicherste Bodennutzung gegen Wassererosion: Zusätzlich zu der permanenten Bedeckung ist mit der Erhöhung der Wurzelmasse eine exponentielle Verringerung der Wassererosion verbunden (Gwendolyn Gyssels et al. 2005). Die Wurzelmasse von Gräsern besteht überwiegend aus Feinwurzeln. Dieser qualitative Unterschied ermöglicht zudem, dass Gräser pro Einheit Bodenvolumen effizienter Wasser (und Nährstoffe) aufnehmen können als Bäume, die über ein so genanntes extensives Wurzelsystem verfügen (Jörg Pfadenhauer/Frank Klötzli 2014, 156, 426; Peter A. H. M. Bakker et al. 2013, 1).

Ob Starkregen oder Dürreperiode: Den entscheidenden Einfluss auf die Wasseraufnahme- und Speicherkapazität der Böden haben die Feinwurzeln - und nicht die reine Wurzelmasse. Die Rhizosphäre des Graslandes verfügt über den weltweit größten Anteil an Feinwurzeln. Sie haben gleichzeitig das größte Bodenbildungspotenzial. Der Fokus auf Bodenfruchtbarkeit und biologische Vielfalt trägt zur Entlastung des Klimas bei. Nachhaltige Beweidung bietet dazu das entscheidende Potenzial (Margret Bunzel-Drüke et al. 22019). Back and forward to the roots ...

\section{Der Klima-Killer ist immer der Mensch-oder: Wir haben es in der Hand}

Es darf keine vorrangige Aufgabe der Landwirtschaft sein, das Klima zu entlasten. Hingegen ist es die vorrangige Aufgabe der Landwirtschaft, die Basisressourcen nachhaltig zu erhalten und zu fördern, um die Ernährung der Weltbevölkerung dauerhaft zu sichern. Indem die Entwicklung der Basisressource Boden gefördert wird, führt das gleichzeitig zur Klimaentlastung. Denn jede zusätzliche Tonne organische Bodensubstanz (Humus) (OBS) entlastet die Atmosphäre um ca. 1,8 Tonnen Kohlendioxid $\left(\mathrm{CO}_{2}\right)$, die sich aus $0,55 \mathrm{t} \mathrm{C}$ und $1,25 \mathrm{t}_{2}$ zusammensetzen. Entsprechend belastet jeder Schwund infolge nicht angemessener Bodennutzung die Atmosphäre mit $\mathrm{CO}_{2}$.

$\mathrm{Zu}$ den großen landwirtschaftlichen Fehlentwicklungen zählen der Umbruch von Grasland bei anschließend nicht-nachhaltigem Ackerbau und 
die zunehmende Fütterung von Grasfressern mit Ackerfrüchten. Mehr als 70 Prozent der in der Landwirtschaft der EU verfütterten Proteine stammen aus Importfutter - insbesondere transgene auf südamerikanischen Äckern mit Glyphosat erzeugte gentechnisch manipulierte Soja. Von Natur aus Weidetiere werden Rinder durch Hochleistungszucht und die Fütterung mit intensiv produziertem Ackerfutter zu Nahrungskonkurrenten der Menschen gemacht.

Hinzu kommt ein grundsätzliches Manko: Nur (Methan-)Emissionen zu berechnen, wird generell allen mit lebendiger Erde verbundenen Systemen nicht gerecht. Denn ihre Klimarelevanz ergibt sich aus der Bilanz Kohlenstoff freisetzender und Kohlenstoff speichernder Prozesse.

Resilienz erfordert eine drastische Reduktion des Fleischkonsums und den Fokus auf Grasland basierte Tierzucht und -haltung/-fütterung. In dem Potenzial, durch nachhaltige Beweidung die ökologischen Basisressourcen zu fördern, liegt zudem eine Voraussetzung für menschliche und tierische Gesundheit.

Landwirtschaft hat nur eine Zukunft, wenn sie in fruchtbaren Landschaften gedacht und behandelt wird. Das erfordert Optimieren statt Maximieren und die Orientierung weg vom kranken Naturbegriff der einseitigen Konkurrenz hin zur biologischen und sozialen Kooperation.

\section{Literatur}

Bakker, Peter A. H. M./Berendsen, Roeland L./Doornbos, Rogier F./Wintermans, Paul C. A. et al. 2013, The rhizosphere revisited: root microbiomics, in: Frontiere in Plant Science 4, 165; DOI: 10.3389/fpls.2013.00165.

Benning, Reinhild/Reichert, Tobias 2016, Vorschläge für eine Neuausrichtung der europäischen Agrarpolitik. Eine Analyse und Position, hg. von Martin Häusling, Wiesbaden; in: https://germanwatch.org/sites/germanwatch.org/files/publication/17687.pdf (abgerufen am 24.03.2020).

BMU - Bundesministerium für Umwelt, Naturschutz und nukleare Sicherheit (Hg.) 2019, Klimaschutz in Zahlen. Fakten, Trends und Impulse, Berlin.

Bülte, Jens 2018, Zur faktischen Straflosigkeit institutionalisierter Agrarkriminalität, in: Goltdammer's Archiv für Strafrecht 165 (1), 35-56.

Bundesverwaltungsgericht 2019, Pressemitteilung Nr. $47 / 2019$ vom 13.06.2019: Töten männlicher Küken tierschutzrechtlich nur noch übergangsweise zulässig, in: https://www.bverwg.de/pm/2019/47 (abgerufen am 14.03.2020).

Bunzel-Drüke, Margret/Reisinger, Edgar et al. 22019, Naturnahe Beweidung und NATURA 2000. Ganzjahresbeweidung im Management von Lebensraumtypen und Arten im europäischen Schutzgebietssystem NATURA 2000, hg. von Arbeitsgemeinschaft Biologischer Umweltschutz, Bad Sassendorf-Lohne. 
Buse, Jörn 2020, Auswirkungen der Parasitenbehandlung bei Weidetieren auf Nicht-Ziel-Organismen am Beispiel von Dungkäfern, in: https://www.naturstift ung-david.de/fileadmin/Medien/Downloads/NNE_Infoportal/Veranstaltungen/2 020-01-21_Tierwohl_in_der_Landschaftspflege/Vortrag_Parasitenbehandlung_b ei_Weidetieren_Buse.pdf (abgerufen am 18.01.2021).

Busse, Tanja 2015, Die Wegwerfkuh. Wie unsere Landwirtschaft Tiere verheizt, Bauern ruiniert, Ressourcen verschwendet und was wir dagegen tun können, Blessing.

Conant, Richard 2010, Challenges and opportunities for carbon sequestration in grassland systems. A technical report on grassland management and climate change mitigation. Plant Production and Protection Division, FAO, Rom.

Dass, Pawlok/Houlton, Benjamin/Wang, Yingping/Warlind, David 2018, Grasslands may be more reliable carbon sinks than forests in California. Environ. Res. Lett.13 074027, in: https://iopscience.iop.org/article/10.1088/1748-9326/aacb39/p df (abgerufen am 17.04.2020).

Eshela, Gidon/Sheponb, Alon/Makovc, Tamar/Milob, Ron 2014, Land, irrigation water, greenhouse gas, and reactive nitrogen burdens of meat, eggs, and dairy production in the United States, in: Proceedings of the National Academy of Sciences USA (PNAS) 111 (33), 11996-12001; DOI: 10.1073/pnas.1402183111.

FAO 2010, Grassland carbon sequestration: management, policy and economics. Proceedings of the Workshop on the role of grassland carbon sequestration in the mitigation of climate change (Rome, April 2009), hg. von Abberton, Michael/Conant, Richard/Batello, Caterina, Rom.

FAO 2014, The State of Food and Agriculture. In brief, Rom.

Fileccia, Turi/Guadagni, Maurizio/Hovhera, Vasyl 2014, Ukraine: Soil fertility to strengthen climate resilience. Preliminary assessment of the potential benefits of conservation agriculture, hg. von FAO/World Bank, Rom.

Gerber, Pierre/MacLeod, Michael/Mottet, Anne/Tempio, Giuseppe et al. 2013, Greenhouse gas emissions from pig and chicken supply chains - A global life cycle assessment. Food and Agriculture Organization of the United Nations (FAO), Rom.

Gerber, Pierre/Steinfeld, Henning/Henderson, Benjamin/Mottet, Anne/Opio, Carolyn/Dijkman, Jeroen/Falcucci, Alessandra/Tempio, Giuseppe 2013, Tackling climate change through livestock. A global assessment of emissions and mitigation opportunities. Food and Agriculture Organization of the United Nations (FAO), Rom.

GRAIN 2019, EU Mercosur Trade Deal will intensify the Climate Crisis from Agriculture. Barcelone, https://www.grain.org/en/article/6355-eu-mercosur-trade-deal -will-intensify-the-climate-crisis-from-agriculture (abgerufen am 25.03.2020).

GRAIN/Institute for Agriculture and Trade Policy (IATP) 2018, Emissions impossible: How big meat and dairy are heating up the planet. Joint publication, Madrid/Minneapolis.

Gyssels, Gwendolyn/Poesen, Jean/Bochet, Esther/Li, Yong 2005, Impact of plant roots on the resistance of soils to erosion by water: A review, in: Physical Geography 29 (2), 189-217; DOI: 10.1191/0309133305pp443ra. 
Hallmann, Caspar A./Sorg, Martin/Jongejans, Eelke/Siepel, Henk et al. 2017, More than 75 percent decline over 27 years in total flying insect biomass in protected areas, in: PLOS ONE 12 (10): e0185809; DOI: org/10.1371/journal.pone.0185809.

Häusling, Martin 2018, Wege aus der Eiweißlücke. Stand und Perspektiven der Eiweißversorgung in der EU, in: Der kritische Agrarbericht 2018, 45-51.

Heubuch, Maria (Hg.) 2019, Der Weltagrarbericht: 10 Jahre danach. Eine kritische Bestandsaufnahme. Wirkung und Folgen des UN-Berichts zur Welternährung und Landwirtschaft, Brüssel; in: https://www.weltagrarbericht.de/fileadmin/files /weltagrarbericht/Neuauflage/Weltagrarbericht10Jahre.pdf (abgerufen am 24.03.2020).

Hörning, Bernhard 2017, Mögliche Auswirkungen der Leistungszucht beim Geflügel auf das Tierwohl. Landwirtschaftskammer Berlin, in: https://www.tieraerzte kammer-berlin.de/images/allgemein/FB_Gefl_2017_12_02-Auswirkungen_der_ Leistungszucht-Hrning.pdf (abgerufen am 14.03.2020).

Idel, Anita 2007, Tötung männlicher Küken: Zweinutzung ist ein Muss, in: Ökologie \& Landbau 142 (2), 30-31.

Idel, Anita 2011, In-Ovo Sexing bei Hühnern, in: https://schweisfurth-stiftung.de/ta g/idel (abgerufen am 14.03.2020).

Idel, Anita 2018, Qualitativer Außenschutz statt schrankenloser Freihandel - für Mensch, Tier und Umwelt, hg. von Kleinbauernvereinigung und Schweizer Tierschutz (STS), Medienkonferenz „Small is beautiful“ (23. März 2018), Bern.

Idel, Anita ${ }^{3} 2019$, Der Wert nachhaltiger Beweidung mit Rind \& Co. für Bodenfruchtbarkeit, Klima und biologische Vielfalt, in: Idel, Anita/Beste, Andrea 32019, Vom Mythos der klimasmarten Landwirtschaft, im Auftrag von Martin Häusling, MdEP/Die Grünen im Europäischen Parlament, Brüssel, 34-73.

Idel, Anita 2020, Zur (Nicht-)Wahrnehmung landwirtschaftlich genutzter Tiere als fühlende Lebewesen: gestern - heute - morgen, in: Schäffer, Johann (Hg.) 2020, Zukunft braucht Vergangenheit. Die Bedeutung der Geschichtsforschung für die Tiermedizin. Freie Themen (20. Jahrestagung der DVG-Fachgruppe Geschichte), Gießen, 173-190.

Idel, Anita ${ }^{8} 2021$, Die Kuh ist kein Klima-Killer! Wie die Agrarindustrie die Erde zerstört und was wir dagegen tun können, Weimar [in Druck].

Idel, Anita/Reichert, Tobias 2013, Livestock production: Livestock production and food security in a context of climate-change and environmental and health challenges, in: UNCTAD (Hg.) 2013, Wake up before it is too late. Transforming Agriculture to cope with climate change and assure food security (Trade and Environment Review 2013), Genf, 137-170.

IPBES - Intergovernmental Science-Policy Platform on Biodiversity and Ecosystem Services 2019, Report of the Plenary of the IPBES on the work of its seventh session, in: https://ipbes.net/sites/default/files/ipbes-7-10_en.pdf (abgerufen am 13.05.2020).

Janjevic, Darko 2019, Thousands of new-born calves illegally killed each year in Germany (Deutsche Welle, 24.11.2019), in: https://p.dw.com/p/3TeDY (abgerufen am 21.04.2020). 
Jürgens, Katrin/Poppinga, Onno/Sperling, Urs 2016, Wirtschaftlichkeit einer Milchviehfütterung ohne oder mit wenig Kraftfutter (Forschungsbericht zur Studie im Auftrag der Internationalen Forschungsgemeinschaft für Umweltschutz und Umwelteinflüsse auf Mensch, Tier, Pflanze und Erde e. V.), Kassel etc.

Karatepe, Ismail Doga/Scherrer, Christoph/Tizzot, Henrique 2020, Das MercosurEU-Abkommen: Freihandel zu Lasten von Umwelt, Klima und Bauern, hg. von Martin Häusling/Die Grünen im Europaparlament, Brüssel.

Klinc, Primoz/Rath, Detlef 2005, Stand und Perspektiven des Einsatzes von durchflusszytometrisch "gesexten" Spermien bei verschiedenen Nutztierarten, in: Züchtungskunde 77 (2/3), 218-229.

Maier, Jürgen 2017, Fluchtursachen - Was können wir bei uns tun? Eröffnungsvortrag, Veranstaltung des Umweltministeriums Niedersachsen, Hannover 28.08.2017, in: https://www.forumue.de/fluchtursachen-was-koennen-wir-bei-un s-tun? (abgerufen am 24.03.2020).

Martens, Holger 2021, Die Milchkuh und Tierwohl: Ein zu lösender Konflikt? in: Neussel, Walter 2021 (Hg.), Verantwortbare Landwirtschaft statt Qualzucht und Qualhaltung. Was warum schief läuft und wie wir es besser machen können, München [in Druck].

McIntyre, Beverly/Herren, Hans/Wakhungu, Judi/Watson, Robert (Hg.) 2009, International Assessment of Agriculture Knowledge, Science and Technology for Development (IAASTD) Global Report, Washington, in: https://www.weltagrar bericht.de/original-berichte.html (abgerufen am 14.03.2020).

Meadows, Donella H./Meadows, Dennis L./Randers, Jørgen/Behrens III, William W. 1972, The Limits to Growth; A Report for the Club of Rome's Project on the Predicament of Mankind, New York.

Milchindustrie-Verband e. V. 2019, Zahlen - Daten - Fakten 2019. Erstellt von ZMB GmbH, Stand: September 2019, in: https://milchindustrie.de/wp-content/ uploads/2018/12/Milchwirtschaft-auf-einem-Blick_1990-2019_Homepage.pdf (abgerufen am 07.05.2020).

Mottet, Anne/Tempio, Giuseppe 2017, Global poultry production: current state and future outlook and challenges, in: World's Poultry Science Journal 73 (2), 245-256; DOI: 10.1017/s0043933917000071.

Necpalova, Magdalena/Lee, Juhwan/Skinner, Colin/Büchi, Lucie et al. 2018, Potentials to mitigate greenhouse gas emissions from Swiss agriculture, in: Agriculture, Ecosystems and Environment 265, 84-102; DOI: org/10.1016/ j.agee.2018.05.013.

Pfadenhauer, Jörg/Klötzli, Frank 2014, Vegetation der Erde. Grundlagen, Ökologie, Verbreitung, Berlin/Heidelberg.

Pimentel, David/Houser, James/Preiss, Erika/White, Omar et al. 1997, Water Resources: Agriculture, the Environment, and Society, in: BioScience 47 (2), 97-106; DOI: 10.2307/1313020.

Pimentel, David/Pimentel, Marcia 2003, World Population, Food, Natural Resources, and Survival, in: World Futures - The Journal of New Paradigm Research 59 (3-4), 145-167; DOI: 10.1080/02604020310124. 
Poeplau, Christopher 2016, Estimating root: shoot ratio and soil carbon inputs in temperate grasslands with the RothC model, in: Plant and Soil 407, 293-305; DOI: $10.1007 / \mathrm{s} 11104-016-3017-3018$.

Probst, Stefan/Wasem, Daniela/Kobel, Desirée/Zehetmeier, Monika/Flury, Christine 2019, Treibhausgasemissionen aus der gekoppelten Milch- und Fleischproduktion in der Schweiz, in: Agrarforschung Schweiz 10 (11-12), 440-445.

Rockström, Johan/Steffen, Will/Noone, Kevin/Persson, Åsa et al. 2009, Planetary boundaries: exploring the safe operating space for humanity, in: Ecology and Society 14 (2), art. 32; online in: http://www.ecologyandsociety.org/vol14/iss2/ar t32/ (abgerufen am 21.04.2020).

Sanders, Jürn/Hess, Jürgen (Hg.) 2019, Leistungen des ökologischen Landbaus für Umwelt und Gesellschaft (Thünen Report 65), Braunschweig; DOI: 10.3220/ REP154704057200.

Sutton, Mark A./Howard, Clare M./Erisman, Jan Willem/Billen, Gilles et al. (Hg.) 2011, The European Nitrogen Assessment. Sources, Effects and Policy Perspectives, Cambridge.

Theile, Merlind 2019, Tierschutz: Diese Küken müssen weg, in: Die Zeit Nr. 25/2019 (13. Juni 2019), in: https://www.zeit.de/2019/25/tierschutz-kueken-t oetung-urteil-bundesverwaltungsgericht (abgerufen am 14.03.2020).

Thompson, Rona L./Lassaletta, Luis/Patra, Prabir K./Wilson, Christopher et al. 2019 , Acceleration of global $\mathrm{N}_{2} \mathrm{O}$ emissions seen from two decades of atmospheric inversion, in: Nature Climate Change 9, 993-998; DOI: 10.1038/ s41558-019-0613-7.

Topping, Christopher J./Aldrich, Annette/Berny, Philippe 2020, Overhaul environmental risk assessment for pesticides. Align regulation with environmental reality and policy, in: Science 367 (6476), 360-363; DOI: 10.1126/science.aay1144.

Umweltbundesamt 2020, Indikator: Grünlandfläche, in: https://www.umweltbund esamt.de/indikator-gruenlandflaeche (abgerufen am 18.01.2021).

Van Wesemael, Dorien/Vandaele, Leen/Ampe, Bart/Cattrysse, Hannes et al. 2019, Reducing enteric methane emissions from dairy cattle: Two ways to supplement 3-nitrooxypropanol, in: Journal of Dairy Science 102 (2), 1780-1787; DOI: 10.3168/jds.2018-14534.

Willner, Wolfgang 2013, Pannonische Steppenrasen in Österreich, in: Baumbach, Henry/Pfützenreuter, Stephan (Hg.) 2013, Steppenlebensräume Europas: Gefährdung, Erhaltungsmaßnahmen und Schutz. Thüringer Ministerium für Landwirtschaft, Forsten, Umwelt und Naturschutz (TMLFUN), Erfurt, 151-162.

Wilstacke, Ludger 2011, Tierhaltung im Spannungsfeld verschiedener Interessen. Unveröffentlichter Vortrag bei der Landwirtschaftstagung der Evangelischen Akademie Villigst et al., Schwerte, 30.11.-01.12.2011.

von Witzke, Harald/Noleppa, Steffen/Zhirkova, Inga ${ }^{4}$ 2014, Fleisch frisst Land, hg. von WWF Deutschland, Berlin.

Wolf, Julie/Asrar, Ghassem/West, Tristram 2017, Revised methane emissions factors and spatially distributed annual carbon fluxes for global livestock, in: Carbon Balance and Management 12, art. 16; DOI: 10.1186/s13021-017-0084-y. 